\title{
Propagation of neutron-reaction uncertainties through multi-physics models of novel LWR's
}

\author{
Augusto Hernandez-Solis ${ }^{\mathrm{a}}$, Henrik Sjöstrand, and Petter Helgesson \\ Uppsala University, Department of Physics and Astronomy, Division of Applied Nuclear Physics, 75120 Uppsala, Sweden
}

\begin{abstract}
The novel design of the renewable boiling water reactor (RBWR) allows a breeding ratio greater than unity and thus, it aims at providing for a self-sustained fuel cycle. The neutron reactions that compose the different microscopic cross-sections and angular distributions are uncertain, so when they are employed in the determination of the spatial distribution of the neutron flux in a nuclear reactor, a methodology should be employed to account for these associated uncertainties. In this work, the Total Monte Carlo (TMC) method is used to propagate the different neutron-reactions (as well as angular distributions) covariances that are part of the TENDL-2014 nuclear data (ND) library. The main objective is to propagate them through coupled neutronic and thermal-hydraulic models in order to assess the uncertainty of important safety parameters related to multi-physics, such as peak cladding temperature along the axial direction of an RBWR fuel assembly. The objective of this study is to quantify the impact that ND covariances of important nuclides such as U-235, U-238, Pu-239 and the thermal scattering of hydrogen in $\mathrm{H} 2 \mathrm{O}$ have in the deterministic safety analysis of novel nuclear reactors designs.
\end{abstract}

\section{Introduction}

\subsection{Background}

A High Conversion Water Reactor (HCWR) or Light Water Breeder Reactor (LWBR) is a nuclear reactor which is cooled by light water and can produce more fissile material than it consumes. In Japan, Hitachi is designing the Resource-renewable Boiling Water Reactor (RBWR) model as part of their innovative water reactor for flexible fuel cycle (FLWR) program [1]. This reactor operates with mixed oxide (MOX) fuel and has a breeding ratio of 1.01. The core is characterized by two fissile zones sandwiched between axial internal blankets of depleted uranium dioxide (UOX). Unlike conventional BWRs, this advanced reactor design is axially heterogeneous with fissile zones producing neutrons and blanket zones consuming them. Therefore, the goal of this design will be to have a LWR that would provide for a self-sustaining fuel cycle in which depleted uranium is used for the makeup fuel. Typically, an RBWR fuel assembly contains five different enrichment fuel pins radially, and axially it contains five distinct active axial core zones: lower blanket, lower fissile, internal blanket, upper fissile and upper blanket. In this type of design, a high axial leakage is needed to keep the void coefficient of reactivity negative. Instead of losing these neutrons, they interact in spatially separate blanket zones to produce more fissile isotopes. The RBWR fuel assembly, as well as its axial configuration are shown in Fig. 1a and 1b, respectively.

\subsection{Motivation}

The motivation of this work came out from ongoing efforts to perform transient analyses for the RBWR. Before

a e-mail: augusto.lehameau@gmail.com any transient calculations are performed, a steady-state solution with a converged power and thermal-hydraulic (TH) distribution is needed. The focus of this work is to employ the best estimate (BE) coupled neutronic and $\mathrm{TH}$ models that have been developed at Uppsala University (UU) in collaboration with Chalmers, and that have resulted in the development of a code known as CORE SIM -TH [2]. The main objective of this paper is to propagate nuclear data covariances through the state variables of the system. Thus, the different uncertainties coming from fission and capture reactions, spectra, as well as energy-angle distributions are meant to be propagated to the final computation of the neutronic and $\mathrm{TH}$ variables of a system comprised of an RBWR single fuel assembly. This is done both under steady-state and transient conditions.

\section{Methodology}

From the development of the TENDL-2012 library, its associated uncertainties are not only found in the respective MF covariance files but are also present in the form of different random files. TENDL-2014 contains different random files for important nuclides, where all the important reactions for U235, U238 and Pu239, as well as the thermal scattering part of $\mathrm{H} 1-\mathrm{H} 20$ have been perturbed. This feature offers a good and pragmatic opportunity to extend the capabilities of CORES SIM $\mathrm{TH}$ in order to perform uncertainty analysis (UA) via the Total Monte Carlo (TMC) method [3,4]. This is possible since after the proper processing of such random files into the common ACE format by means of the NJOY code, the computation of the energy-collapsed and homogenized macroscopic cross-sections can be performed with the Monte Carlo SERPENT code. From thereafter, such

(C) The Authors, published by EDP Sciences. This is an Open Access article distributed under the terms of the Creative Commons Attribution License 4.0 (http://creativecommons.org/licenses/by/4.0/). 


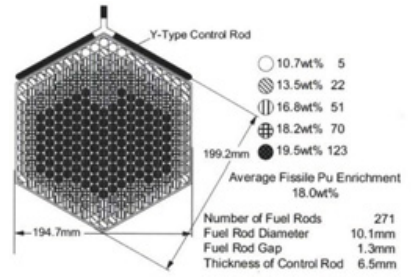

(a) Hexagonal Fuel Assembly Configuration

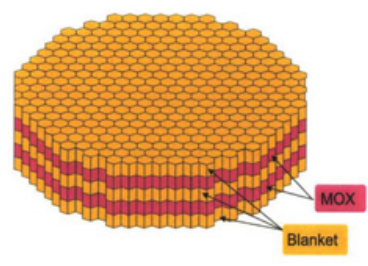

(b) Axial Zone Configuration in Full Core Design
Figure 1. RBWR fuel assembly and core layout [1].

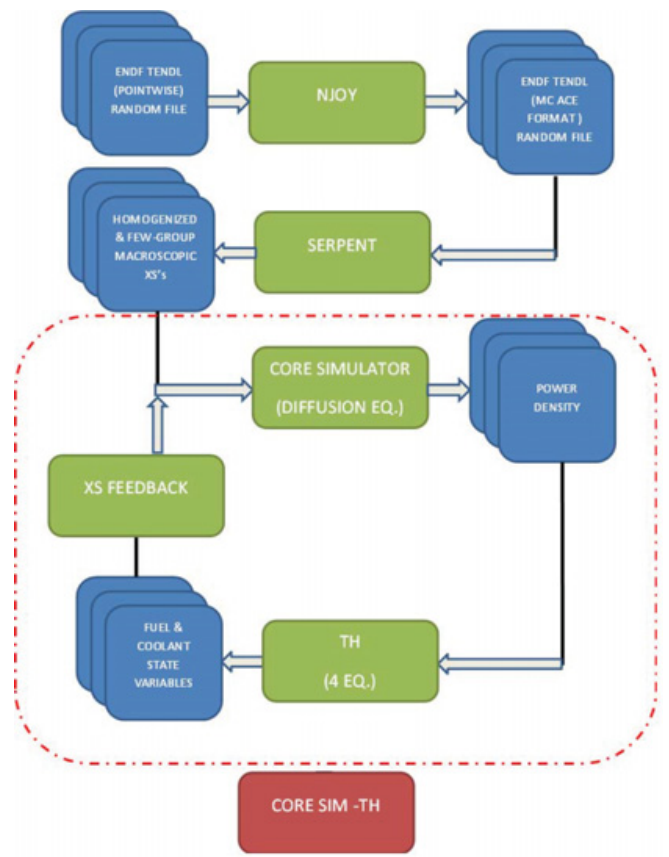

Figure 2. TMC applied to CORE SIM - TH for the UA of an RBWR assembly.

parameters are ready to be used as inputs to CORE SIM $-\mathrm{TH}$. If this procedure is applied to each random file, then it is possible to assess and quantify the uncertainty in the nodal state-variables of the system under study. This methodology is depicted below in Fig. 2.

Under steady-state, the feedback is done by updating the different nodal cross-sections due to changes in fuel temperature and coolant density, utilizing an interpolation scheme from a database created from different branches. More info about the nodal coupled neutronic and TH model of CORE SIM-TH under steady-state conditions can be found in [5].

\section{Study case}

\subsection{Steady-state}

100 random files were computed in order to apply the TMC method to CORE SIM - TH for the UA of an RBWR fuel assembly. Thus, both neutronic and $\mathrm{TH}$ variables along the axial length are computed 100 times in order to show the spread of the UA. Following the use of Wilk's formula [6], 100 calculations would be enough to cover $95 \%$ of the population with a $95 \%$ of confidence for a single variable like for instance, the assembly keffective. Some steadystate results are shown in Fig. 3.
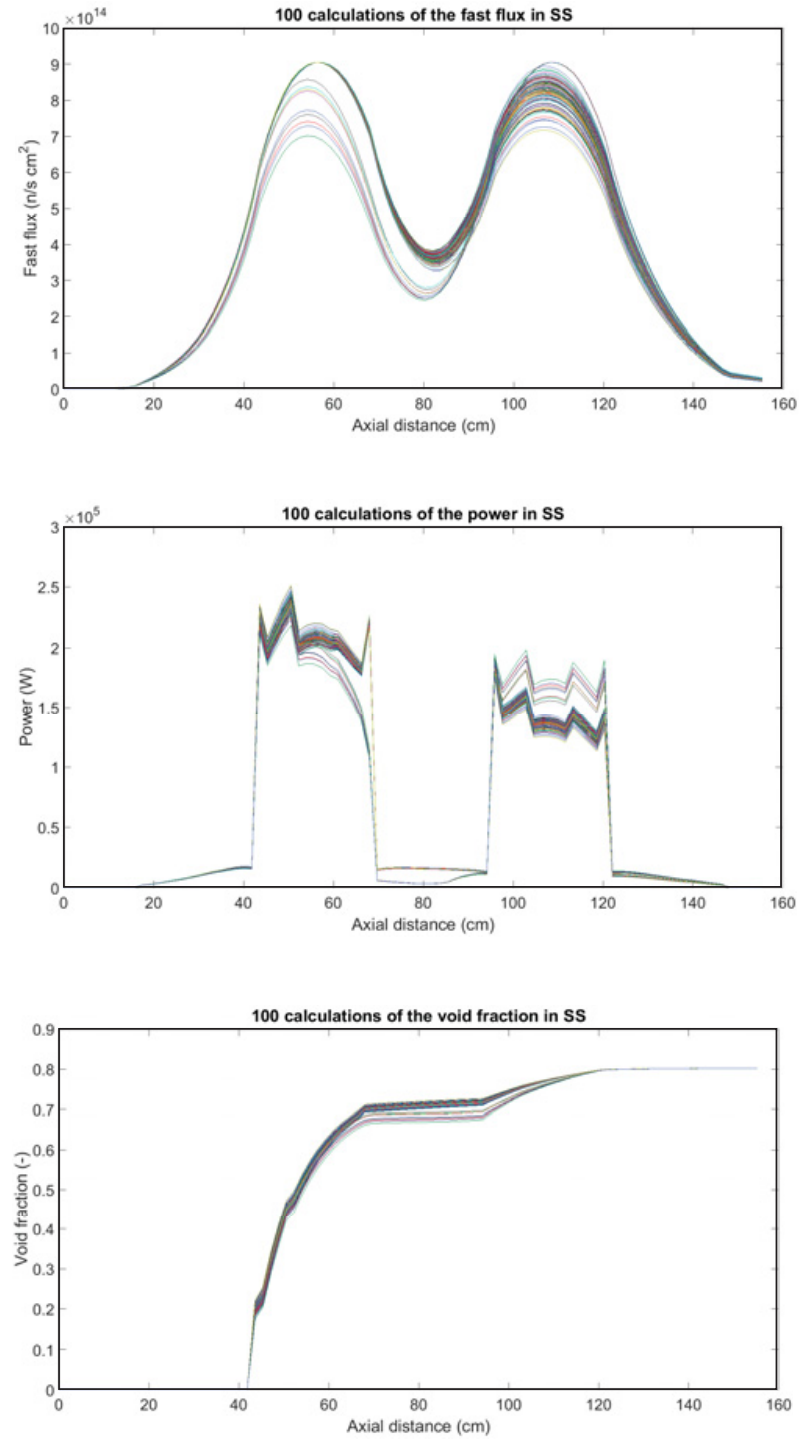

Figure 3. Steady-state results of the UA.

\subsection{Transient}

A transient case corresponding to the modeling of a control rod $(\mathrm{CR})$ withdrawal has been applied to the TMC scheme of CORE SIM - TH. This is done for a time period of 2 seconds with a common time step of 0.05 seconds. Such transient modeling has been imposed by changing the thermal fission and fast absorption cross-sections at each node, and following a linear relationship as a function of the axial direction. Thus, it is assumed that there is no feedback in this study as a function of time, and that the estimation of the uncertainty of the different $\mathrm{TH}$ parameters are in open loop.

After 100 calculations, the mean value of the coupled parameters can be found below both as a function of time and space. Therefore, the relative uncertainty estimated such as:

$$
\Delta P=\frac{\operatorname{std}(P)}{\operatorname{mean}(P)} \times 100(\%)
$$

can also be evaluated as a function of time and space as well for the different estimated parameters, as it can be seen in Fig. 4 for the assembly axial power. The aim of this analysis is just to make a first attempt to see how 

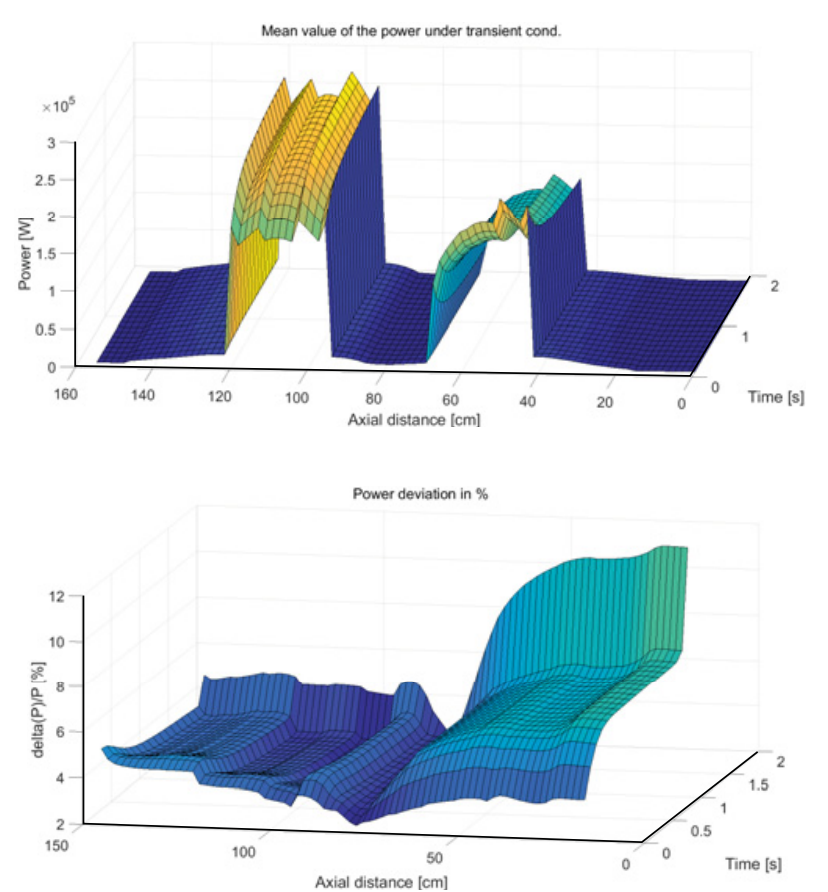

Figure 4. UA under transient conditions of the assembly power after 100 runs.

the different nuclear data random files impact the multiphysics modeling even if it is in open loop.

\section{Conclusions}

This paper reports results of an uncertainty analysis technique, such as the TMC method, being applied to the BE deterministic analysis of the novel RBWR design. The modeling scheme of CORE SIM - TH allows the proper propagation of nuclear data uncertainty through the different multi-physics stages under steady-state. On the other hand, a much better modeling of the transient scenario is required, where the time changes of the different nodal cross-sections physically agree with the neutronics and TH feedback. In this paper, a first approach to test the time-dependent capabilities of the TMC computational platform was just being tested. Therefore, one important task that could be planned for the future is the modeling of time-dependent feedback scenarios.

Due to recent development in computational resources, and the ongoing efforts to evaluate covariances of neutronreactions and angular distributions in modern nuclear data libraries such as TENDL-2014, it is nowadays possible to propagate such uncertainties and properly evaluate its impact on important safety parameters related to the multiphysics phenomena that is present in nuclear reactors.

\section{References}

[1] Takeda, R., Miwa, J., and Moriya, K. (2007). BWRs for Long-Term Energy Supply and for Fissioning almost all Transuraniums. In Proc. Global 2007, Boise, Idaho

[2] Hernandez-Solis, A, Demaziere, C. (2015). Development of an in-house coupled neutronic and thermalhydraulic code for the steady-state analysis of LWRs. In the proceedings of $16^{\text {th }}$ Reactor Physics Meeting of the Nordic Countires, Göteborg, Sweden

[3] Sjöstrand, H., Alhassan, E., Duan, J., Gustavsson, C., Koning, A. et al., Propagation of nuclear data uncertainties for ELECTRA burn-up calculations. Nuclear Data Sheets 118, 527-530 (2014)

[4] Koning A.J., Rochman D., Modern Nuclear Data Evaluation with the TALYS Code System. Nuclear Data Sheets 113(12), 2841-2934 (2012)

[5] Demaziere, C., Dykin, V., Hernandez-Solis, A., Development of three-dimensional capabilities for modelling stationary fluctuations in nuclear reactor cores. Annals of Nuclear Energy 84, 19-30 (2015)

[6] Wilks, S.S., Determination of sample size for setting tolerance limits. Annals of Mathematical Statistics 13, 91-96 (1941) 